$1.035-0,1311 \mathrm{~B}=1.000$, c'est-à-dire $\mathrm{B}=266,9$ ou bien à : $1.000+0,1311 \mathrm{~B}_{\mathrm{k}}=1.035$, c'est-à-dire $\mathrm{B}_{\mathrm{k}}=266,9$.

Ces deux matières grasses (au litre ou au kilogramme) s'expriment l'une en fonction de l'autre par une courbe qui est un arc d'hyperbole. La différence d'ordonnée entre la valeur de $B_{k}$ ou de $\mathrm{B}$ et la bissectrice de l'angle des coordonnées donne, en tout point, la différence numérique entre ces deux valeurs (voir graphique $\mathrm{n}^{0} 5$ ).

Nous espérons que quelques-unes de ces formules, ainsi que les tableaux et les graphiques qu'elles ont permis d'établir, pourront rendre certains services dans la pratique courante de plusieurs industries.

\title{
REVUE
}

\section{LES PROBLÈMES QUI RESTENT A RÉSOUdRE DANS L'INDUSTRIE LAITIÈRE}

\author{
par \\ G. GÉNIN \\ Ingénieur E. P. C.
}

A l'occasion de la Conférence technologique des produits alimentaires qui a eu lieu au "Massachusetts Institute of Technology ", en septembre 1937, et dont nous avons donné un court compte rendu, M. H. S. JudKINS, vice-président des "Sealtest System Laboratories ", a présenté une communication sur les problèmes qui restent encore à résoudre dans l'industrie laitière dont nous avons extrait les lignes qui suivent.

Les problèmes qui se posent dans l'industrie laitière apparaissent dès la production du lait à la ferme. Le lait est produit dans des millions de fermes ayant des caractéristiques très diverses, depuis la très petite installation qui ne dispose que de quelques pâturages, jusqu'aux fermes spécialisées qui sont de véritables industries. Dès la production, se posent tout d'abord certaines difficultés qui ont trait au problème de la production économique du lait, de l'obtention d'un lait de bonne qualité et de l'utilisation des excédents de lait non eonsommables sur place et qu'il faut par conséquent ramasser dans un grand nombre d'établissements dispersés.

Aux Etats-Unis, le Ministère de l'Agriculture subventionne un certain nombre d'associations de producteurs de lait dont le but est d'arriver à améliorer la production du lait à la ferme, mais le nombre 
de fermes qui appartiennent à ces associations est encore aujourd'hui très réduit et on peut dire que la production d'un animal est encore actuellement celle qu'elle était il y à 10 ans, e'est-à-dire d'environ 4.000 pounds par année (environ $1.800 \mathrm{~kg}$.). Or, étant donné les conditions économiques qui règnent aux Etats-Unis, cette production n'atteint même pas la moitié de celle qu'il faudrait obtenir pour que la production du lait soit une opération réellement rentable. Il est toujours vrai de dire que la quantité de lait produit dans beaucoup de fermes eouvre à peine le prix de la nourriture distribuée aux animaux.

D'importants progrès ont été réalisés d'autre part dans l'amélioration de la qualité du lait, mais il reste encore beaucoup à faire, en particulier auprès des petits propriétaires de quelques vaches seulement. Les règles imposées par les municipalités d'un grand nombre de villes obligent le vendeur de lait à distribuer un lait sain et propre. On peut déjà regretter qu'il n'y ait pas une plus grande uniformité entre les différents règlements ainsi édictés. On sait aujourd'hui quelles sont les précautions qu'il faut prendre pour obtenir du lait sain, propre, de bon goût; il suffit donc de porter à la connaissance des producteurs les précautions qu'ils doivent prendre, pour obtenir ce résultat.

Le fait que la production de lait est beaucoup plus importante en été qu'en hiver, alors qu'au contraire la consommation est à peu près uniforme d'un bout de l'année à l'autre, pose le problème de l'utilisation de l'excédent du lait et de l'uniformisation des cours de ce produit. Les différents plans que l'on a proposés pour codifier les conditions d'achat du lait sont trop nombreux pour être exposés ici et le Gouvernement s'est même intéressé à la question, cherchant à réaliser un programme d'achat du lait qui soit satisfaisant pour tous. La plupart de ees programmes repose sur l'idée d'accorder aux producteurs un prix déterminé pour sa production de base et de lui accorder un autre prix pour le surplus de la production qu'il pourra vendre. On prévoit également d'accorder des primes suivant la qualité du lait et suivant l'usage qui en est fait. Généralement, les cours du lait sont fixés soit par de grandes sociétés laitières qui font le ramassage, soit à la suite d'accords entre l'industriel et les syndicats agricoles.

D'importants progrès ont été réalisés dans la question du transport du lait dans des wagons et camions-citernes isolés. Un autre progrès plus récent, qui semble appelé à un certain avenir, est l'emploi de petits réservoirs isolés que l'on peut placer sur des remorques ou sur des wagons et qui assurent le transport du lait depuis la production jusqu'á l'usine d'utilisation, en évitant toute manipulation de ce produit si sensible. 
Dans l'outillage des laiteries, il faut également citer d'importants progrès réalisés à la suite de l'emploi de l'acier inoxydable pour la construction des appareils. Il est certain que des progrès plus importants encore pourront être réalisés lorsque l'industrie laitière pourra coopérer d'une façon encore plus étroite avec les constructeurs de matériel et d'outillage. Il y a encore beaucoup à faire dans certains domaines, comme par exemple dans la construction de l'outillage pour la fabrication du petit-lait, la manipulation de la crème et la simplification des méthodes de pasteurisation.

L'essai à la phosphatase employé maintenant pour déterminer si la pasteurisation du lait a été effectuée correctement a pu être vérifié à maintes reprises dans diverses usines et semble appelé à un avenir important.

Jusqu'à ces dernières années, on n'avait pas enregistré d'importantes modifications en ce qui concerne la bouteille à lait et ses conditions de transport et de livraison. La question des bouteilles est d'une grande importance, étant donné les dépenses considérables auxquelles on est conduit par leur emploi, surtout dans le commerce de gros où on ne peut disposer de bouteilles en location. On a donc apporté beaucoup d'intérêt à l'apparition des bouteilles en papier paraffiné, mais dans ce domaine, les améliorations restent à faire.

La crème est un important produit de l'industrie laitière et il faut encore apporter à sa préparation beaucoup d'améliorations si l'on veut obtenir un produit qui soit parfaitement uniforme, qui au bout d'un certain temps ne se sépare pas en petit-lait et en amas de matières grasses plus ou moins coagulées et qui donne, lors de la fabrication de la crème fouettée, toujours d'excellents résultats.

Enfin, le problème des différents goûts défectueux du lait qui apparaissent principalement en hiver est un de ceux dont la complexité est très grande, et constamment des travaux sont publiés sur ce sujet montrant les causes et les façons de remédier à ces défauts.

\section{Lait condensé.}

Le lait condensé qui est du lait réduit dans la proportion de 2 à 1 a $v u$ ses applications se multiplier, en particulier dans le domaine de la cuisine. C'est ainsi qu'aux Etats-Unis, de 1920 à 1936, la consommation de ce produit a triplé. L'écart qui existe entre le prix du lait concentré et celui du lait normal est souvent assez élevé, du fait que le lait qui sert à la préparation du lait eoncentré provient généralement de régions très éloignées des villes. Il est également regrettable de constater que souvent le lait brut employé pour cette fabrication n'est pas aussi parfait que le lait vendu dans les villes soumis à des conditions de contrôle très sévères. Cette 
question du contrôle du lait utilisé par l'industrie est une de celles qui se pose avec acuité et on cherche également à produire un lait concentré qui, par dilution, donnerait un produit absolument analogue, au point de vue coloration et goût, au lait normal.

\section{Ice-cream.}

Les progrès dans lá fabrication de la crème glacée ont été très rapides. Ils ont porté en premier lieu sur la construction des réfrigérants, ce qui a permis de remplacer les anciens appareils à glace et sel par des appareils à expansion ou à saumure réfrigérante. On est également parvenu à construire des appareils permettant de fabriquer d'une façon continue la crème glacée. D'un côté de l'appareil, on fait pénétrer un courant constant de mélange pour crème glacée et de parfums alimentaires et de l'autre côté, on recueille une crème glacée prête à être consommée à la température d'environ $-5^{\circ}$.

Toutefois, il reste encore à réaliser certains progrès dans la construction du matériel. Pour ce qui est de la préparation des mélanges, on étudie actuellement l'influence de la nature et de la qualité des constituants de la crème sur sa texture et sur sa saveur, et on s'efforce en particulier, de faire disparaître le défaut.souvent constaté d'un goût de carton dans la crème.

En ce qui concerne plus particulièrement le choix et le traitement des fruits qui sont très fréquemment ajoutés aux mélanges, ainsi que le choix des parfums alimentaires, on effectue actuellement d'importantes études, car ces produits étant ajoutés après pasteurisation, il peut en résulter une augmentation du nombre des bactéries et des $B$. Coli contenus dans la crème.

Les spécialistes sont d'ailleurs loin d'être d'accord sur les caractéristiques des meilleures qualités de crème glacée, en ce qui concerne particulièrement leur teneur en graisse et leur texture. La crème glacée étant surtout livrée aux consommateurs par de petits détaillants, la question s'est posée d'éviter qu'il subsiste chez ces commerçants des stocks de crème perdant leur fraîcheur. On * également étudié les conditions à remplir pour que la crème glacés soit fournie aux consommateurs dans des conditions d'hygiène parfaite et on étudie actuellement la température la plus favorable pour la conservation et la distribution de la crème glacée.

\section{Fromages.}

A la suite des progrès apportés à la fabrication des fromages aux Etats-Unis, et devant le développement des diverses variétés de fromages et en même temps de leurs applications, la consommation aux Etats-Unis a augmenté et atteint aujourd'hui près de 
2 kg. 5 par habitant et par an. Toutefois, il ne faut pas s'arrêter à ce résultat et on peut espérer que cette consommation atteindra celle qu'on enregistre dans certains pays européens. C'est ainsi qu'en Suisse par exemple, la consommation atteint près de 8 kilogrammes par habitant et par an. Une consommation de cette importance n'a pu être obtenue que par des recherches approfondies en vue d'améliorer la qualité, la conservation, et de trouver d'autres types plaisant à la clientèle.

\section{Problèmes divers.}

Pour terminer, nous mentionnerons encore quelques problèmes qui se posent aux directeurs de laiteries. En premier lieu, le choix et la préparation de produits destinés au nettoyage et à la stérilisation des appareils. Ce problème est rendu particulièrement complexe du fait des caractéristiques très variables de l'eau dont on dispose dans les différentes régions.

On étudie également la question du traitement des résidus des laiteries qui, dans les régions agricoles où on ne trouve pas d'installations d'épuration des eaux, présente une grande importance. Il n'a pas encore été possible de trouver jusqu'aujourd'hui un procédé réellement satisfaisant d'épuration des eaux résiduaires.

\section{BIBLIOGRAPHIE ANALYTIQLE}

\section{JOURNAUX, REVUES, SOCIÉTÉS SAVANTES PRODUCTION, HYGIENE}

Guttonneau (G.), Mocquot (G.) et Eyrard (A.). - Sur la microfiore envahissante des laits de consommation, son origine et sa signification dans les contrôles hygiéniques. Bulletin de l'Académie de Médecine, t. CXVIII, 21 décembze 1937, no 39 . p. 754 .

Dans cette note, les A, ont voulu préeiser les eonditions de l'invasion microbienne des laits de consommation et l'interpréter.

Le milieu utilisé pour la numération bactérienne a été le suivant : milieu gélosé au lait écrémé, soumis aseptiquement à l'aetion de la trypsine, pendant un temps limité (1); l'incubation des plaques se faisant à $30^{\circ}$.

Les A. ayant distingué la contamination initiale et la contamination secondaire, montrent que la première est inhérente à la production même et qu'elle est sans conséquence pratique pour l'altération des laits. Au contraire, la contamination secondaire, qui se produit après la récolte du lait, porte très vite la teneur microbienne du liquide à plusieurs millions ou dizaines de millions de germes au $\mathrm{cm}^{3}$. Les germes de cette contamination secondaire sont

(1) Voir Guittonneau, Sajods et De Peet, Le Lait, 10, 1930, p. 750. 